\title{
Beurteilung konkurrierender Interessen bei Leitlinien zur Behandlung von Substanzstörungen
}

\author{
Methodik und Ergebnisse
}

\author{
Gerhard Bühringer und Robert Czernecka \\ Institut für Psychologie und Psychotherapie, Technische Universität Dresden
}

\begin{abstract}
Zusammenfassung: Hintergrund: Ab 2018/2019 wurden drei S3-Leitlinien (LL) zur Behandlung von alkohol-, medikamenten- und tabakbezogenen Störungen entwickelt und verabschiedet. Neben dem fachlich korrekten Vorgehen bei der Entwicklung von LL sind die Erfassung und Bewertung möglicher konkurrierender Interessen (KI) der Teilnehmenden zentral für die Qualität der LL. Zielsetzung: Beschreibung der Methodik bei der Bearbeitung möglicher KI und der Ergebnisse. Methodik: Zu 11 Themenbereichen möglicher materieller und immaterieller KI von 217 Teilnehmenden wurden die Angaben mit einem vorgegebenen Fragebogen, teilweise vorgegebenen Kriterien, einem neu entwickelten Kodierungssystem für die Angaben zu möglichen KI sowie einem neu entwickelten System für Beteiligungseinschränkungen nach fachlichen Themenbereichen der LL ausgewertet. Ergebnisse: 69 von 217 Teilnehmenden (32\%) hatten keinen KI, 48 (22\%) einen KI mit geringer Relevanz (limitierte Leitungsfunktion), 86 (40\%) mit moderater (Stimmenthaltung), weitere 14 (6\%) mit hoher Relevanz (Beratungsausschluss). Durch das neue Instrument themenbezogener Mitwirkungsbegrenzungen konnten sich dennoch von den 148 Mitwirkenden mit einer Einschränkung weitere $60 \%$ bis $90 \%$ bei einzelnen Themen beteiligen. Schlussfolgerungen: Das Beurteilungssystem fand eine hohe Akzeptanz, bei nur etwa $5 \%$ war eine zweite Überprüfung notwendig. Die statistischen Kennziffern unterscheiden sich erheblich zwischen verschiedenen LL, und erfordern methodenkritische Studien und bessere Instruktionen mit Beispielen, insbesondere zu immateriellen KI.
\end{abstract}

Schlüsselwörter: Konkurrierende Interessen, Leitlinien, Substanzstörungen, Methodik

Evaluation of Competing Interests in Guidelines for Treating Substance Use Disorders: Methods and Outcome

\begin{abstract}
Background: Since 2018/2019, three S3 treatment guidelines (GL) for alcohol-, pharmaceutical-, and tobacco-related disorders have been developed and approved in Germany. In addition to the scientifically correct procedures for developing GL, the collection and evaluation of potentially competing interests $(\mathrm{Cl})$ are central to the quality of the $\mathrm{GL}$. Aims: Description of methods for processing potential $\mathrm{Cl}$ and of results. Methods: Data on 11 themes concerning potential financial and non-financial $\mathrm{Cl}$ from 217 participants have been collected using an existing questionnaire. Analysis took place by partly drawing on existing criteria, partly utilizing a newly developed coding system for information on potential $\mathrm{Cl}$, as well as a newly developed system of topic-related voting limitations. Findings: 69 participants ( $32 \%)$ had no $\mathrm{Cl}, 48(22 \%)$ had a $\mathrm{Cl}$ with limited relevance (leading position), 86 (40\%) had a moderate level $\mathrm{Cl}$ (abstention from voting) and an additional 14 (6\%) had a high-level $\mathrm{Cl}$ (exclusion from session). Through the application of the new system of topic-related voting limitations, it was possible for $60 \%$ to $90 \%$ of the 148 contributors with a limitation to still participate, depending on the GL topic. Conclusions: The assessment system for potential Cl and related exclusion measures reached a high level of acceptance; a re-evaluation was necessary in only about $5 \%$ of cases. The statistical indicators differed considerably between the different GL. This requires further methodological analyses and better instructions using examples, particularly with regards to non-financial $\mathrm{Cl}$.
\end{abstract}

Keywords: Competing interests, guidelines, substance use disorders, methods

\section{Einführung}

Dieser Beitrag befasst sich mit der Methodik zur Einstufung möglicher konkurrierender Interessen bei den Teilnehmenden an der Entwicklung der S3-Leitlinien für Screening, Diagnose und Behandlung von Alkoholbezogenen Störungen (AWMF, 2021a), Rauchen und Tabakabhän- gigkeit (AWMF, 2021b), und Medikamentenbezogenen Störungen (AWMF, 2020a) sowie deren Ergebnissen. Die Deutsche Gesellschaft für Suchtforschung und Suchttherapie (DG-Sucht) hat die Leitung und Koordination in einer Steuerungsgruppe zusammen mit der Deutschen Gesellschaft für Psychiatrie, Psychotherapie, Psychosomatik und Nervenheilkunde (DGPPN) übernommen und die drei 
Leitlinien (LL) von 2018 bis 2021 mit Expert_innen aus den jeweiligen Themenbereichen entwickelt. Der gesamte Prozess erfolgte mit Unterstützung und nach den Standards der Arbeitsgemeinschaft der Wissenschaftlichen Medizinischen Fachgesellschaften (AWMF), u.a. festgehalten im Dokument „Deutsches Instrument zur methodischen Leitlinien-Bewertung" (DELBI; AWMF \& äzq, 2008). Die LL Medikamente gilt seit 08.2020, die beiden anderen seit 01.2021, jeweils für fünf Jahre. Für alkoholbezogene Störungen (AWMF, 2015; Mann, Hoch \& Batra, 2015) und Tabak (Batra et al., 2016) gab es bereits Vorgängerversionen.

LL zur Behandlung sind in den letzten Jahren zu einem wichtigen Instrument der Qualitätssicherung im Gesundheitsbereich geworden. Sie sind vor allem für Praktiker_innen eine Grundlage für die aktuelle Umsetzung der jeweiligen wissenschaftlichen Erkenntnisse und der fachlichen Konsensbildung in therapeutisches Handeln. Sie bilden auch einen Rahmen für fachliche Kontroversen oder juristische Auseinandersetzungen (Friese \& Wienke, 2020). Zentral für die Qualität der LL sind neben der wissenschaftlich korrekten Evidenzrecherche und der Auswahl fachlich kompetenter Mitglieder der Leitliniengruppen ihre neutrale und unbeeinflusste Einschätzung der jeweiligen Evidenz und der Transformation der Erkenntnisse in die einzelnen LL. Einige spektakuläre Fälle im Bereich manipulierter wissenschaftlicher Aussagen im Tabak- und Pharmabereich (Grüning, Gilmore \& McKee, 2006; Hoogendoorn \& de Lange, 2013; WHO, nach 2004) haben u. a. dazu geführt, die Teilnehmenden an LL Entwicklungen kritischer als früher auf mögliche problematische Beeinflussungen ihrer unabhängigen wissenschaftlichen Beurteilung zu überprüfen, d.h., inwieweit wissenschaftsfremde Interessen die Beurteilung des Kenntnisstandes und die Leitlinienformulierung beeinflussen könnten.

Bei den zitierten Fällen, z.B. im Tabakbereich, ging es um kriminelles Verhalten. Viel wichtiger -und Gegenstand dieses Beitrags- sind aber die transparente Darstellung und Handhabung möglicher ,alltäglicher" konkurrierender Interessen (KI), die sich aus der Finanzierung der Forschung, der Zugehörigkeit zu interessengeleiteten Organisationen oder Arbeitgebern oder aus eigenen fachlichen Interessen ergeben können. In der „Erklärung von Interessen und Umgang mit Interessenkonflikten bei Leitlinienvorhaben" (AWMF, 2018) wird folgendes zu KI festgehalten (Seite 1):

„Interessenkonflikte sind definiert als Gegebenheiten, die ein Risiko dafür schaffen, dass professionelles Urteilsvermögen oder Handeln, welches sich auf ein primäres Interesse bezieht, durch ein sekundäres Interesse unangemessen beeinflusst wird. Interessenkonflikte sind nicht per se negativ zu bewerten. Sie manifestieren sich durch das Nebeneinander von primären Interessen (z. B. bei Leitlinienautoren die Formulierung evidenzund konsensbasierter Empfehlungen zur Verbesserung der Ver- sorgungsqualität) und sekundären Interessen (z.B. direkte und indirekte finanzielle, akademische, klinische, persönliche), deren Ausprägungsgrade und Bedeutungen variieren können. Interessenkonflikte sind somit oft unvermeidbar, aber nicht zwangsläufig problematisch im Hinblick auf eine Beeinflussung der Leitlinieninhalte. Entscheidend für die Legitimation und Glaubwürdigkeit von Leitlinien in der Wahrnehmung durch Öffentlichkeit und Politik sind nach internationaler Auffassung Transparenz und der faire, vernünftige Umgang mit Interessenkonflikten"

(siehe Guidelines International Network, 2012).

Vier wichtige Aspekte sind dabei relevant:

- Erstens geht es dabei nicht um eine moralische Bewertung guter oder schlechter Interessen, sondern um einen Prozess der Transparenz möglicher Faktoren, die ein unabhängiges Urteil beeinflussen können. Sekundäre Interessen zu dem vorrangigen primären Interesse an wissenschaftlich basierten Aussagen und Beurteilungen treten immer auf und können nicht vermieden werden. Sie sind nicht per se negativ oder zwangsläufig als problematisch zu bewerten, können aber eine konkurrierende Funktion zu primären Interessen haben. Eine leitende Vertretung eines Vereins zur Förderung medikamentengestützter Therapie kann ein sehr ehrenwertes Engagement darstellen, gleichzeitig aber auch eine Quelle möglicher konkurrierender Interessen bei der Beurteilung von Therapieoptionen sein. Zur Vermeidung von moralischen Bewertungen wird international statt von Interessenskonflikten (conflict of Interest, COI) zunehmend von konkurrierenden Interessen (KI) gesprochen (competing interests, CI).

- Zweitens geht es nicht um die subjektive Sicht der Betroffenen, sondern um die Einschätzung neutraler Dritter. Subjektiv kann man sich selbst als frei von Interessenskonflikten wahrnehmen, objektiv wird aber die Finanzierung von Studien aus dem Alkoholwirtschaft bei neutralen und unabhängigen Dritten möglicherweise zu einer abweichenden Einschätzung führen.

- Drittens haben neben den traditionell im Vordergrund stehenden Firmen als Arbeitgeber oder Geldgeber für Forschung auch gewerbliche und gemeinnützige Einrichtungen und Verbände und staatliche Institutionen finanzielle und nichtfinanzielle Interessen, und entsprechende Verbindungen von Teilnehmenden an Leitlinien erfordern die gleiche Aufmerksamkeit und Deklaration.

- Viertens geht es nicht nur um konkurrierende Interessen finanzieller Art, sondern auch um immaterielle weltanschauliche, politische oder fachpolitische Interessen ohne (direkte) finanzielle Relevanz, die ein unabhängiges Urteil beeinflussen können und eine Deklaration erfordern (The PLoS Medicine Editors, 2008). 
Es gibt inzwischen für die Deklaration und Beurteilung von Interessen sowie möglicher Einschränkungen bei der Mitwirkung an Entscheidungen, Gutachten und Leitlinien eine Vielzahl von Regelungen von Fachgesellschaften (Society for the Study of Addiction, nach 2012; AWMF 2020b), Fachzeitschriften (z.B. ADDICTION, 2020; SUCHT, 2020) und internationalen Fachorganisationen (COPE, 2020; Guidelines International Network, 2012 ISAJE, 2019). Trotz mehrjährigerer Anwendung solcher Regelsysteme besteht wenig Übereinstimmung über (a) die jeweiligen Informationen dazu, was deklariert werden soll, (b) die jeweiligen Regeln für die Einstufung von KI sowie (c) die Schlussfolgerungen für Einschränkungen der Mitwirkung an der LL Entwicklung, es gibt keine gemeinsamen Regelungen.

\section{Zielsetzung}

Ziel des Beitrags ist die Darstellung der Erfassung von KI bei den Beteiligten und der Einstufung für mögliche Einschränkungen bei der Mitwirkung an der Erstellung der drei LL. Neben der Vorstellung der Ergebnisse, stehen die Beschreibung der Methodik und eine ausführliche Diskussion methodischer Aspekte im Vordergrund.

\section{Methodik}

An der Entwicklung der drei LL beteiligten sich etwa 220 Expert_innen in unterschiedlichen Funktionen als Autor_ innen, Gutachter_innen und Koordinator_innen.

\section{Erhebungsinstrument}

Die Teilnehmenden für die LL Medikamente hatten das von der AWMF vorgegebene Formular ,Erklärung von Interessen" in der Betaversion vom 29.06.2016 ausgefüllt, zumeist im ersten Halbjahr 2018. Die Angaben aus den Formularen wurden vom Ärztlichen Zentrum für Qualität in der Medizin (äzq) in eine Excel Tabelle übertragen. Die Angaben in diesen Tabellen waren Grundlage der Bewertung möglicher KI durch die Autoren, so dass die Validität dieser Angaben nicht geprüft werden konnte. Die Einträge der Teilnehmenden für die LL Alkohol und Tabak erfolgten zumeist 2019 in einer aktualisierten Version des AWMF Formulars vom 23.05.2018, die den Autoren zur direkten Auswertung vorlagen (AWMF, 2018).

In beiden Fragebogen werden der Arbeitgeber der letzten drei Jahre und die Position bzw. Funktion in der Insti- tution erfragt, sowie die Funktion in der Leitliniengruppe und der Zeitraum, auf den sich die Erklärung bezieht. Arbeitgeber/Institution und die Position/Funktion wurden bei der Beurteilung indirekter (materieller und nichtmaterieller) KI berücksichtigt. Bei den direkten Interessen werden über das laufende Jahr der Erhebung und weitere drei Jahre zuvor Informationen zu sechs Bereichen erfasst: Berater-/Gutachtertätigkeit, Mitarbeit in einem Wissenschaftlichen Beirat (advisory board), Vortrags-/ oder Schulungstätigkeit, Autoren-/oder Co-Autorenschaft, Forschungsvorhaben/Durchführung klinischer Studien sowie Eigentümerinteressen (Patent, Urheberrecht, Aktienbesitz). $\mathrm{Zu}$ jedem Bereich folgen Fragen nach: Kooperationspartner, Zeitraum der Beziehung, Bezug zur Leitlinie, Art und Höhe der Zuwendung, Empfänger (persönlich oder Institution).

Die indirekten Interessen erfassen fünf Themenbereiche: Mitgliedschaft/Funktion in Interessenverbänden, Schwerpunkte wissenschaftlicher Tätigkeiten, Publikationen, Schwerpunkte klinischer Tätigkeiten, Federführende Beteiligung an Fortbildungen/Ausbildungsinstituten, Persönliche Beziehungen (als Partner oder Verwandte_r 1. Grades zu einem Vertretungsberechtigten eines Unternehmens der Gesundheitswirtschaft). Zusätzlich werden erfasst: Zeitraum und Themenbezug zur LL. Zuletzt können Sonstige Interessen angegeben werden, „die von Dritten als einschränkend in Bezug auf Ihre Objektivität oder Unabhängigkeit wahrgenommen werden könnten“.

Die beiden Versionen des Fragebogens enthalten gleiche Hinweise zur Nutzung der Daten und zu ihrer Aufbewahrung: Die Originale verbleiben demnach vertraulich beim Projektkoordinator. Standardisierte Zusammenfassungen der Inhalte, das Verfahren der Sammlung und Bewertung der Informationen sowie die Ergebnisse der Diskussion zum Umgang mit KI sind in der Dokumentation zur Entwicklung der LL offenzulegen. Am Ende der beiden Fragebogen bestätigen die Teilnehmenden mit Ihrer Unterschrift und mit fast gleichen Formulierungen, dass sie alle relevanten Informationen aufgeführt haben, dass sie Diskussionen zur Erklärung anderer Teilnehmenden absolut vertraulich behandeln, und dass die persönlichen Angaben in einer standardisierten Zusammenfassung im Leitlinienreport veröffentlicht werden. Die beauftrage Person für die Auswertung und Beurteilung der KI sowie ein Mitarbeiter haben Vertraulichkeitserklärungen unterschrieben, und in den drei Berichten zur Auswertung und Einstufung möglicher KI sind die eigene Deklaration zu den Themen der LL eingefügt, ausgeweitet auf verwandte Bereiche aller Abhängigkeitserkrankungen.

Die beiden Versionen unterscheiden sich nur in zwei Punkten, nämlich zum einen in einer Frage -zusätzlich zur Art der Zuwendung- zu ihrer Höhe, die nur in der Fassung 
von 2018 enthalten ist. Weiterhin enthält die neuere Version vom 23.05.2018 Informationen zur DSGVO.

\section{Gewichtung möglicher konkurrierender Interessen}

Die Einstufungen der Angaben nach der Ausprägung eines KI und den damit verbundenen Einschränkungen bei der Mitwirkung an der Entwicklung der LL erfolgte gemäß den AWMF Vorgaben (AWMF, 2020b):

- KI [0]: kein konkurrierendes Interesse

- KI [1-0]: konkurrierendes Interesse ohne Bezug zur aktuellen LL

- KI [1-1]: konkurrierendes Interesse mit Bezug zur aktuellen LL, von geringer Relevanz (Limitierung von Leitungsfunktionen)

- KI [1-2]: konkurrierendes Interesse mit Bezug zur aktuellen LL, von moderater Relevanz (Stimmenthaltung)

- KI [1-3]: konkurrierendes Interesse mit Bezug zur aktuellen LL, von hoher Relevanz (Ausschluss von Beratungen).

Zusätzlich wurde wegen der umfassenden Themenbereiche der LL, von Diagnostik über Therapie zu Versorgungstrukturen, ein System zur weiteren Differenzierung der KI [1-1] bis [1-3] entwickelt, das die jeweiligen Einschränkungen auf Themenbereiche begrenzt. Eine Person kann dabei für mehrere Themenbereiche deklariert und in ihren Mitwirkungsrechten eingeschränkt werden, und auch für unterschiedliche Stufen, z.B. bei einem Themenbereich einer LL ein moderater KI mit Stimmausschluss, bei einem anderen Themenbereich der gleichen LL ein hochrelevanter KI mit Beratungsausschluss:

- LL zur Zuordnung der Behandlung zu bestimmten Einrichtungssystemen oder -strukturen, z.B. für leitende Vertreter von Suchtverbänden, Psychotherapie- oder Psychiatrieverbänden oder Berufsverbänden, weiterhin leitende Mitarbeiter von Einrichtungstypen wie psychiatrische, sozialtherapeutische oder Suchteinrichtungen (1)

- LL zur Durchführung der Behandlung in ambulanten oder stationären Einrichtungen, z. B. für Vertreter eines Verbandes für ambulante Suchteinrichtungen, niedergelassene Ärzte und Psychotherapeuten oder stationäre Psychiatrien (2)

- LL zum Einsatz von Psychotherapie (3)

- LL zum Einsatz von Pharmakotherapie (4)

- LL zum Einsatz von sozialen/sozialtherapeutischen Maßnahmen (5).

\section{Einstufung möglicher konkurrierender Interessen}

Die „Kriterien zur Einschätzung der Relevanz“ der AWMF umfassen zwei zentrale Dimensionen (AWMF, 2020b, Algorithmus): (1) Ausprägung der Sekundärinteressen (Art der Zuwendung, Höhe der Zuwendung und Empfänger) sowie (2) Ausmaß des Konflikts (Art der Beziehung/Tätigkeit, Zeitraum der Beziehung/Tätigkeit und Kooperationspartner). Diese Vorgaben der AWMF geben zwar eine Orientierung, sind aber weitgehend unpräzise. Deshalb wurde von den Autoren aufgrund früherer Erfahrungen bei der Beurteilung von KI bei der LL Entwicklung folgendes System von Kriterien für die Einstufung entwickelt.

\section{Direkte finanzielle Interessen}

Jede finanzielle Zuwendung in einer der fünf Kategorien des Fragebogens führte zu der Einstufung eines vorläufigen KI [1].

- Ist diese Zuwendung/Beauftragung ohne Bezug bzw. gibt es zeitlich und/oder häufigkeitsmäßig nur einmalige oder ganz wenige Anlässe, wurde ein nicht relevantes KI [1-0] vergeben, in anderen Fällen ein relevantes KI.

- Die Einstufung der Stärke der Relevanz eines KI in gering [1-1], moderat [1-2] oder hoch [1-3] erfolgte nach der Höhe und Häufigkeit der Zuwendungen. Die Einstufung [1-3] war nur für eine dauerhafte finanzielle $\mathrm{Zu}$ wendung in mehreren der fünf Themenbereiche von LL vorgesehen.

- Hatten die finanziellen Zuwendungen eine klare thematische Begrenzung, wurde ein KI auf einen oder mehrere der genannten fünf Themenbereiche von LL eingeschränkt.

\section{Indirekte Interessen}

Jede Angabe in den fünf Kategorien oder bei „Sonstiges“, die auf ein berufliches, wissenschaftliches, praxisbezogenes, ausbildungsbezogenes oder privates Interesse hinweist, das die unabhängige wissenschaftliche Beurteilung von Sachverhalten einschränken könnte, führte zu der automatischen Einstufung eines vorläufigen KI [1].

- Einfache Mitgliedschaften in Berufsverbänden mit einem gerichteten Interesse wurden mit einem nicht relevanten KI [1-0] eingestuft,

- leitendende Funktionen in solchen Berufsverbänden mit einem KI [1-1],

- leitendende Funktionen in einem Berufsverband mit einem der nachfolgend genannten verbandlichen Interesse an der Art des Behandlungssettings bzw. der Art der Behandlung wurden mit einem KI [1-2] eingestuft: Suchtverbände, Verbände zur Förderung ambulanter oder stationärer Behandlung, Verbände mit einem Interesse an der Unterstützung psychotherapeutischer oder 
pharmakotherapeutischer Behandlung oder eines besonderen Interesses an sozialen Maßnahmen.

- aktive regelmäßige Tätigkeit in einem Aus-/Fortbildungsinstitut mit einem bestimmten Interesse (z.B. Psychotherapie) führte zu einem KI [1-1], leitende Mitarbeit zu einem KI [1-2].

\section{Durchführung der Einstufung}

Nach einer Entwicklung des Rasters wurden in einem ersten Durchlauf alle Teilnehmenden der LL Entwicklung eingestuft. Aufgrund der Erfahrungen dieses Durchlaufs wurden das Raster verfeinert und mit einem Abstand von einigen Tagen alle Teilnehmenden in einem zweiten Durchlauf erneut geprüft und bei Bedarf die Einstufungen modifiziert. Bei unklaren Informationen erfolgte eine unabhängige Einstufung durch den Zweitautor dieses Artikels und anschließende Diskussion der Einschätzungen bis zu einem Konsens.

Die jeweiligen Angaben aus den Formularen (LL Alkohol und Tabak) bzw. der Übersicht (Medikamente) wurden vom Autor nach den vorstehenden Kriterien bewertet und die Beurteilung in eine Tabelle „Tabellarische Übersicht Interessenskonflikte" mit folgenden Spalten übergeführt: Name, Unterschriftsdatum des Formulars durch Teilnehmer_in, Arbeitgeber_in/Vertretende Organisation, Funktion in der vertretenden Institution, die sechs Themenbereiche zu direkten Interessen, die fünf Bereiche zu indirekten Interessen, Sonstiges, sowie das Gesamtergebnis der Beurteilung. Bei unklaren Angaben erfolgten in begrenztem Umfang zusätzliche Recherchen im Internet. Diese waren zeitaufwendig, erbrachten aber in unklaren Fällen hilfreiche Informationen. Fragebögen mit unzureichenden bzw. unleserlichen Angaben wurden zur weiteren Ergänzung zurückgegeben. Nachzügler und Nachrücker wurden zu einem späteren Zeitpunkt, gelegentlich Monate später, nachbeurteilt. Personen, die an beiden, im gleichen Zeitraum stattfindenden LL Konferenzen Alkohol und Tabak teilnahmen, wurden getrennt ausgewertet, und eigenständig in Bezug auf die jeweilige LL Konferenz beurteilt, da grundsätzlich unterschiedliche Sachverhalte bestehen konnten. Da der Einstufungsund Beurteilungsprozess trotz aller Präzisierungsversuche Ermessensspielräume offen lässt, hatten alle Teilnehmenden die Möglichkeit, bei Eindruck einer Fehleinschätzung Rücksprache mit dem Beurteilenden zu halten.

\section{Ergebnisse}

Insgesamt wurden bei 217 Teilnehmenden der drei LL die Angaben zu ihren KI ausgewertet, dazu kommen die Personen, die bereits in der Vorbereitungs- oder Anfangszeit ausgeschieden sind (Tabelle 1). Der Anteil der Teilnehmenden ohne (relevanten) KI lag für Alkohol und Medikamente nur bei etwa $25 \%$, bei Tabak bei fast $50 \%$. Auffällig ist mit etwa $40 \%$ der Anteil der Teilnehmenden mit moderater Relevanz, der nach AWMF Vorgabe zum Ausschluss bei Abstimmungen führt, und weitere $6 \%$ mit einer hohen Relevanz, die laut Vorgaben von den Beratungen auszuschließen wären. Dazu kommen $22 \%$ mit einer geringen Relevanz (Limitierung bei Leitungsfunktionen).

Tabelle 1. Ergebnisse der Auswertungen möglicher konkurrierender Interessen der Mitglieder der S3 LL Gruppen „Medikamenten bezogene Störungen“" ,Alkohol bezogene Störungen“ und „Störungen durch Tabakkonsum“

\begin{tabular}{|c|c|c|c|c|}
\hline Einstufung & $\begin{array}{l}\text { S3 LL „Medikamenten } \\
\text { bezogene Störungen“ }\end{array}$ & $\begin{array}{l}\text { S3 LL „Alkohol } \\
\text { bezogene Störungen“ }\end{array}$ & $\begin{array}{l}\text { S3 LL „Störungen } \\
\text { durch Tabakkonsum“ }\end{array}$ & Summe \\
\hline $\begin{array}{l}\text { KI }[1-0] \\
\text { kein oder konkurrierendes Interesse ohne Bezug } \\
\text { zur aktuellen LL }\end{array}$ & $16(25 \%)$ & $19(24 \%)$ & $34(47 \%)$ & $69(32 \%)$ \\
\hline $\begin{array}{l}\text { KI [1-1] konkurrierendes Interesse mit Bezug zur } \\
\text { aktuellen LL, von geringer Relevanz } \\
\text { Limitierung Leitungsfunktion }\end{array}$ & $21(32 \%)$ & $17(21 \%)$ & $10(14 \%)$ & $48(22 \%)$ \\
\hline $\begin{array}{l}\text { KI [1-2] konkurrierendes Interesse mit Bezug zur } \\
\text { aktuellen LL, von moderater Relevanz } \\
\text { Stimmenthaltung }\end{array}$ & $28(43 \%)$ & $37(46 \%)$ & $21(29 \%)$ & $86(40 \%)$ \\
\hline $\begin{array}{l}\text { KI [1-3] konkurrierendes Interesse mit Bezug zur } \\
\text { aktuellen LL, von hoher Relevanz } \\
\text { Ausschluss von Beratungen }\end{array}$ & $0(0 \%)$ & $7(9 \%)$ & $7(10 \%)$ & $14(6 \%)$ \\
\hline Gesamtanzahl & $65(100 \%)$ & $80(100 \%)$ & $72(100 \%)$ & $217(100 \%)$ \\
\hline
\end{tabular}


Um den hohen Ausschluss von fast $70 \%$ zu vermeiden, wurden die Einschränkungen jeweils auf die Themenbereiche einer LL begrenzt, bei denen ein konkreter KI vorlag. Zum besseren Verständnis sind in Tabelle 2 die Angaben zu den Teilnehmenden mit bereichsbeschränkten Mitwirkungsrechten für fünf definierte Themenbereiche der LL angegeben: In den Kopfzeilen stehen die Gesamtwerte für die Personen mit einer Einschränkung, darunter jeweils die Zahl der Personen mit einer Mitwirkung für einen eingeschränkten Bereich. Mit dieser selektiven Beteiligung an einzelnen Bereichen der LL war es möglich, dass je nach Thema $60 \%$ bis $90 \%$ der mit KI deklarierten Teilnehmenden mitwirken konnten, so dass sich z.B. der gesamte Anteil der Abstimmungsberechtigten (KI [1-1] und KI [1-2]) von $54 \%$ je nach Thema auf $75 \%$ bis $95 \%$ erhöhte.

Ein Problem war der hohe anfängliche Anteil von etwa 101 Fragebögen (47\%) mit unvollständigen Angaben für die Auswertung, bedingt durch die Konstruktion des Fragebogens, der es bei einer fehlenden Angabe nicht ermög- lichte zwischen nicht zutreffend und vergessen auszufüllen zu unterscheiden. Fehlende Werte wurden nacherhoben, und alle betroffenen Auswertungen wiederholt. Von den 217 Teilnehmenden haben nur etwa 10 Personen eine Nachbeurteilung wegen fehlender Akzeptanz der ersten Einstufung erbeten. Diese führte in etwa der Hälfte der Fälle zu einer Korrektur.

\section{Zusammenfassung und Diskussion}

Im ersten Teil werden Methodik und Ergebnisse diskutiert und einige Verbesserungsvorschläge entwickelt, im zweiten Teil folgen einige allgemeine Überlegungen $\mathrm{zu}$ KI und ihrer Deklaration. Es wurden die deklarierten KI von 217 Teilnehmenden ausgewertet, davon hätten nach der Auswertung 86 (40\%) nicht abstimmen und 14 (6\%) nicht an Beratungen teilnehmen können, insgesamt fast

Tabelle 2. Auszug aus Tabelle 1 mit Angaben zu den themenbezogenen Mitwirkungsrechten der Teilnehmenden der LL Gruppen mit einer Beschränkung gemäß KI [1-1] bis KI [1-3], 69 Teilnehmende (32\%) ohne KI nicht aufgeführt

\begin{tabular}{|c|c|c|c|c|}
\hline & $\begin{array}{l}\text { S3 LL „Medikamenten } \\
\text { bezogene Störungen“ }\end{array}$ & $\begin{array}{l}\text { S3 LL „Alkohol } \\
\text { bezogene Störungen“ }\end{array}$ & $\begin{array}{l}\text { S3 LL „Störungen } \\
\text { durch Tabakkonsum“ }\end{array}$ & Summe \\
\hline $\begin{array}{l}\text { KI [1-1] (Limitierung } \\
\text { Leitungsfunktion) }\end{array}$ & 21 & 17 & 10 & $48(22 \%)$ \\
\hline (1) Zuordnung zu Behandlungseinrichtungen/-systemen & 13 & 15 & 7 & 35 \\
\hline (2) Frage ambulante /stationäre Behandlung & 5 & 16 & 9 & 30 \\
\hline (3) Einsatz von Psychotherapie/Verhaltenstherapie & 11 & 16 & 9 & 36 \\
\hline (4) Einsatz von Pharmakotherapie & 7 & 15 & 7 & 29 \\
\hline (5) Einsatz sozialer /sozialtherapeutischer Maßnahmen & 21 & 17 & 9 & 47 \\
\hline KI [1-2] (Stimmenthaltung) & 28 & 37 & 21 & $86(40 \%)$ \\
\hline (1) Zuordnung zu Behandlungseinrichtungen/-systemen & 5 & 23 & 17 & 45 \\
\hline (2) Frage ambulante /stationäre Behandlung & 24 & 33 & 19 & 76 \\
\hline (3) Einsatz von Psychotherapie/Verhaltenstherapie & 24 & 22 & 9 & 55 \\
\hline (4) Einsatz von Pharmakotherapie & 21 & 34 & 18 & 73 \\
\hline (5) Einsatz sozialer /sozialtherapeutischer Maßnahmen & 26 & 33 & 18 & 77 \\
\hline KI [1-3] (Ausschluss) & 0 & 7 & 7 & $14(6 \%)$ \\
\hline (1) Zuordnung zu Behandlungseinrichtungen/-systemen & 0 & 3 & 4 & 7 \\
\hline (2) Frage ambulante/stationäre Behandlung & 0 & 5 & 5 & 10 \\
\hline (3) Einsatz von Psychotherapie/Verhaltenstherapie & 0 & 5 & 4 & 9 \\
\hline (4) Einsatz von Pharmakotherapie & 0 & 6 & 6 & 12 \\
\hline (5) Einsatz sozialer /sozialtherapeutischer Maßnahmen & 0 & 7 & 7 & 14 \\
\hline
\end{tabular}


die Hälfte. Dazu kamen 48 Personen (22\%) mit einer Limitierung bei Leitungsfunktionen. Durch die Anwendung eines von den Autoren eingeführten Systems begrenzter Teilnahmebeschränkungen auf einzelne Themenbereiche der LL war es möglich, die Zahl der Beteiligungsbeschränkungen von fast $70 \%$ auf je nach Themenbereich $10 \%$ bis $40 \%$ zu reduzieren, oder umgekehrt konnten z.B. statt $54 \%$ jetzt $75 \%$ bis $95 \%$ an Abstimmungen teilnehmen. Aufwendig war die hohe Zahl von Wiederholungsbeurteilungen wegen fehlender Hinweise im Fragebogen zur Kategorie „,nicht zutreffend“. Das Einstufungssystem und die Ergebnisse fanden eine gute Akzeptanz, nur etwa $5 \%$ baten um eine erneute Beurteilung, die bei der Hälfte zu einem korrigierten Ergebnis führte. Nach Rückmeldung der Leitlinienkoordinatoren für alle drei LL wurden die Ergebnisse der Einstufungen und die sich daraus ergebenden Einschränkungen (Limitierung bei Leitungsfunktionen, Stimmenthaltung oder Ausschluss von Beratungen) bei der Entwicklung und Abstimmung umgesetzt.

$46 \%$ ohne Abstimmungsberechtigung (KI mit moderater oder hoher Relevanz) würde zur Beschlussunfähigkeit führen, bzw. zumindest zu einer aufwendigen Suche und Beurteilung neuer Teilnehmenden. Uns erschien dieser Anteil hoch, allerdings haben wir keine systematische Übersicht über die Ergebnisse bei anderen Leitlinien gefunden. In einer Publikation zur Aktualisierung der S3-Leitlinie „Langzeitanwendung von Opioiden bei nicht-tumorbedingten Schmerzen - LONTS“ (Häuser, Klose, Welsch, Petzke \& Nothacker, 2020) wurden von 37 Teilnehmenden nur 5 mit einem geringen oder mäßigen KI eingestuft, also ein fast 10-fach geringerer Anteil. Durch die themenbereichsbezogenen Einschränkungen bei der Mitwirkung konnte zwar bei den berichteten LL eine gute Mitwirkungsrate erreicht werden, die ausgesprochen hohen Unterschiede bei der Einstufung der KI zwischen LL sollten allerding systematisch untersucht werden, um diese -soweit sie methodische Artefakte sind- einzuschränken, und gleichzeitig den Beurteilungsprozess zu optimieren.

Zum Fragebogen in der aktuellen Fassung von 2018 gibt es als Erfahrung aus dem Auswertungsprozess zahlreiche Verbesserungsvorschläge. Ein struktureller Vorschlag wurde bereits umgesetzt, die Fragebogen können auch online ausgefüllt werden (AWMF, 2018), damit entfallen „kryptoanalytische“ Kompetenzen beim Entziffern einiger handschriftlich ausgefüllter Fragebogen und das Problem der Erkennung fehlender bzw. nichtzutreffender Antworten. Das Online-Ausfüllen der Fragebogen sollte noch stärker forciert werden. Inhaltlich sollten folgende Vorschläge geprüft werden:

- Instruktionspsychologische Optimierung der Erläuterungen und des Fragebogens zur Reduzierung von Missverständnissen und Nachfragen, die detailliert auf die vier zentralen Themen bei der Deklaration möglicher KI eingehen, die in der Einleitung genannt wurden: (1) neutrale Einstufung, keine moralische Bewertung, (2) Beurteilung durch Dritte ist relevant, nicht subjektive Einschätzung, (3) nicht nur Firmen haben ökonomische Interessen, auch (gemeinnützige) Verbände und Behörden, (4) immaterielle Interessen sind schwierig zu erfassen, aber ebenso wichtig.

- Neben der Differenzierung finanzielle/immaterielle Interessen sollten die Begriffe direkt (finanziell)/indirekt (immateriell) aufgegeben werden, da die Immateriellen auch sehr direkte Interessen betreffen können.

- Zur Reduzierung des Aufwandes die Einfügung einer „Bagatelle“-Obergrenze von Zahlungen pro Bezugsjahr für die sechs relevanten Bereiche, bis zu der auf Angaben verzichtet werden kann, z. B. tausend oder zweitausend Euro.

- Bessere Kriterien für Angaben zum Empfänger von Zahlungen, da auch ohne Entscheidungsverantwortlichkeit finanzielle Vorteile entstehen können.

Der Missbrauch wissenschaftlicher Reputation zur „Begründung" von Falschaussagen aus ökonomischen Interessen wie bei der Beurteilung der Gefährlichkeit des Tabakrauchens (Grüning, Gilmore \& McKee, 2006; WHO, nach 2004) hat in der Vergangenheit zu einem großen Misstrauen gegenüber interessengeleiteter Finanzierung wissenschaftlicher Aktivitäten geführt, und als Gegenreaktion zur heutigen Praxis der sorgfältigen Deklaration solcher Interessen. Allerdings hätten heutige Deklarationspflichten das damalige, als kriminell einzustufende Verhalten nicht verhindert, und sie verhindern auch heute nicht die immer wieder aufgedeckten Beispiele wissenschaftlichen Fehlverhaltens durch bewusste Verfälschung von Evidenz. Bewusste Fälschung kann man durch die Deklaration von KI nicht verhindern. Insofern ist es auch wichtig solche strafbaren Sachverhalte von der Bewertung der KI zu trennen: Der Wettbewerb unterschiedlicher und entgegengesetzter Interessen ist in einer pluralistischen demokratischen Gesellschaft grundsätzlich als legitim und wünschenswert anerkannt (von Brünneck, 2007) und erfordert die Fähigkeit zu permanenten Diskussionen und Kompromissen. Grenzen findet ein Wettbewerb dann, wenn ein Interesse durch finanzielle Mittel und Macht (materiell) oder gesellschaftliche Macht (immateriell) Bewertungen und Entscheidungen maßgeblich beeinflusst. Dies festzustellen und zu verhindern ist auch das Ziel der Deklaration von Interessen in unserem Bereich der Entwicklung von LL, ihrer unabhängigen Bewertung sowie der Festlegung möglicher Einschränkungen bei der Mitwirkung an LL. Um die Akzeptanz des gesamten Prozesses in der Wissenschaft zu fördern, ist somit eine neutrale und transparente Erfassung, Einstufung 
und Bewertung ohne moralische Beurteilungskategorien unerlässlich.

Konkurrierende Interessen wurden traditionell nur bei gewerblichen Geldgebern gesehen. Nur allmählich setzt sich durch, dass auch Verbände - legitime - ökonomische Interessen haben können, zumeist gar nicht für sich selbst als (gemeinnützige) Organisation, aber für ihre Mitgliedsorganisationen, z.B. das Interesse von Fachkliniken bei LL-Entscheidungen zur stationären Behandlung. Und auch Behörden können ausgeprägte Interessen haben: Bereits das zweite Mal hat das Bundesministerium für Finanzen aus Sorge vor einer zu starken Reduzierung der Steuereinnahmen die einmalige Steuererhöhung der Tabaksteuer, von der man weiß, dass sie zu gesundheitlichen Vorteilen durch eine eher stärkere Konsumreduktion geführt hätte, durch eine über viele Jahre verteilte Erhöhung mit gestuften Beträgen durchgesetzt (Spiegel Online, 2003; Süddeutsche Zeitung, 2021). Dass die Einnahmen dem Bundeshaushalt und damit allen Bürgern zugutekommen, bedeutet nicht, dass keine KI von Geld und Gesundheit bestehen, oder es sich um moralisch bessere KI handelt. Und auch die KI bei immateriellen Sachverhalten sind nicht grundsätzlich als weniger bedeutsam einzustufen, wenn man die mächtige und dominante Rolle der DHS und einiger Bundesländer bei der jahrelangen dogmatischen Vertretung des Abstinenzgebots bei Opioidabhängigen betrachtet.

Zum Abschluss noch einige Gedanken zur Begriffsbildung. Der von der AWMF immer noch verwendete Begriff „Interessenskonflikt“ birgt die Gefahr von Missverständnissen aufgrund einer subjektiv erlebten $\mathrm{Zu}$ schreibung der Bewertung durch das betroffene Individuum und der Ablehnung, wenn ein Betroffener selbst keine Konflikte erkennt. Konkurrierende Interessen trifft den Sachverhalt besser, und hat auch keine wertenden Konnotationen.

Ganz korrekt wäre „mögliche konkurrierende Interessen", da das fachliche Handeln der Teilnehmenden an der Entwicklung von LL möglicherweise tatsächlich keiner Konkurrenz zwischen dem wissenschaftlichen Urteil und ökonomisch oder gesellschaftspolitisch bedingten sachfremden Interessen unterliegt. Das konkurrierende Interesse liegt zunächst bei den Geldgebern, die neben der (zumeist zweckgebundenen) Forschungsförderung noch andere Interessen haben, die teilweise nicht konkurrierend sein müssen (Imagebildung, Unterstützung bei der Beurteilung und Bewältigung neuer Probleme, Wettbewerbsvorteile bei neuen Erkenntnissen und Angeboten), aber teilweise konkurrierend zur wissenschaftlichen Evidenz sein können (Abschwächung von Hinweisen auf Wirkungslosigkeit oder gefährliche Nebenwirkungen, sachfremde Betonung von Vorteilen einer bestimmten Therapieform). Dieses komplexe Gebilde möglicher Inter- essen neben der Evidenz ist schwer zu differenzieren. Zum Schutz der Teilnehmenden an LL vor einer kritischen Nähe zu Firmen, Verbänden oder Behörden sowie als Schutz der gesamten Gesellschaft sind deshalb transparente Deklarationen und Bewertungen von möglichen KI durch unabhängige Dritte unerlässlich und sollten ausgebaut werden.

\section{Literatur}

Addiction (2020). Instruction for Authors - 3. Ethical Principles. [Webseite]. Verfügbar unter: https://www.addictionjournal.org/ about-us/ethical-policy [15.02.2021].

AWMF (2015). S3-Leitlinie „Screening, Diagnose und Behandlung alkoholbezogener Störungen”, AWMF-Register Nr. 076-001 LEITLINIENREPORT (Stand: 23.02.2015). [Dokument im Internet]. Verfügbar unter: https://www.awmf.org/leitlinien/detail/ ll/076-001.html [13.02.2021].

AWMF (2018). Erklärung von Interessen [Dokument im Internet]. Verfügbar unter: https://www.awmf.org/fileadmin/user_upload/Leitlinien/AWMF-Regelwerk/Anhaenge/20180523_ AWMF_Formular_Erkl\%C3 \%A4rung_Interessen_V.2.5.docx [04.11.2019].

AWMF (nach 2018). Interessenerklärung Online [Webseite]. Verfügbar unter: https://interessenerklaerung-online.awmf.org/ 13.02.2021].

AWMF (2020a). S3-LeitlinieMedikamentenbezogene Störungen, Langfassung 1. Auflage, 2020 - Version 1.1, AWMF-RegisterNr.: 038-025. [Dokument im Internet]. Verfügbar unter: https:// www.awmf.org/leitlinien/detail/ll/038-025.html [13.02.2021].

AWMF (2020b). AWMF-Regelwerk Leitlinien: Erklärung von Interessen und Umgang mit Interessenkonflikten. [Webseite]. Verfügbar unter: https://www.awmf.org/leitlinien/awmf-regelwerk/ll-entwicklung/awmf-regelwerk-01-planung-und-orga nisation/po-interessenkonflikte.html [13.02.2021].

AWMF (2021a). S3-Leitlinie,,Screening, Diagnose und Behandlung alkoholbezogener Störungen". AWMF-Register Nr. 076-001. [Dokument im Internet]. Verfügbar unter: https://www.awmf. org/leitlinien/detail/ll/076-001.html [13.02.2021].

AWMF (2021b). S3-Leitlinie "Rauchen und Tabakabhängigkeit: Screening, Diagnostik und Behandlung", Langversion, AWMFRegister Nr. 076-006. [Dokument im Internet]. Verfügbar unter: https://www.awmf.org/leitlinien/detail/ll/076-006.html [13.02.2021].

AWMF und äzq (2008). Deutsches Instrument zur methodischen Leitlinien-Bewertung (DELBI). [Dokument im Internet]. Verfügbar unter: https://www.leitlinien.de/mdb/edocs/pdf/litera tur/delbi-fassung-2005-2006-domaene-8-2008.pdf [13.02.2021].

Batra, A., Petersen, K. U., Hoch, E., Andreas, S., Bartsch, G., Gohlke, H. et al. (2016). S3-Leitlinie "Screening, Diagnostik und Behandlung des schädlichen und abhängigen Tabakkonsums”. Kurzfassung. SUCHT 62(3), 125-138.

von Brünneck (2007). Ernst Fraenkel - Gesammelte Schriften, Band 5. Demokratie und Pluralismus. Baden-Baden: Nomos.

COPE (2020). Conflicts of interest /Competing interests [Webseite]. Verfügbar unter: https://publicationethics.org/competinginterests [15.02.2021].

Friese, K., V. \& Wienke, A. (2020). Facharztstandard und Leitlinien im Arzthaftungsrecht. HNO 68, 944-945. https://doi.org/10. 1007/s00106-020-00881-6 
Grüning, T., Gilmore, A. B. \& McKee, M. (2006). Tobacco industry influence on science and scientists in Germany. Am J Public Health, 96(1), 20-32. doi:10.2105/ajph.2004.061507

Guidelines International Network (2012). Toward International Standards for Clinical Practice Guidelines. Annals of Internal Medicine, 156(7), 525-531. doi:10.7326/0003-4819-156-7$201204030-00009 \%$ m 22473437

Häuser, W., Klose, P., Welsch, P., Petzke, F. \& Nothacker, M. (2020). Leitlinienreport der zweiten Aktualisierung der S3-Leitlinie "Langzeitanwendung von Opioiden bei nicht-tumorbedingten Schmerzen - LONTS“. Schmerz, 34(3), 245-278. doi: 10.1007/ s00482-020-00471-z.

Hoogendoorn, M.-L. \& de Lange, A. (2013). Scientific misconduct in the pharmaceutical industry. Social Cosmos, 4 (1), 34 - 39. URN:NBN:NL:UI:10-1-114220

ISAJE (2019). Statements and Guidelines Ethical Guidelines. [Webseite]. Verfügbar unter: http://www.isaje.net/ethical-guidelines.html [01.11. 2019].

Mann, K., Hoch, E. \& Batra, A. (2015) S3-Leitlinie. S3-Leitlinie Screening, Diagnose und Behandlung alkoholbezogener Störungen. Heidelberg: Springer.

Society for the study of Addiction (nach 2012). Ethical Policy - Editorial Independence. [Webseite]. Verfügbar unter: https://www. addictionjournal.org/guidance/instructions-for-authors\#ethi cal-principles [15.02.2021].

Spiegel Online (2003). Regierung will Raucher bei der Stange halten - Die Packung Zigaretten wird um einen Euro teurer, aber nicht auf einen Schlag. Um die Mehreinnahmen zu sichern, hat Bundesfinanzminister Eichel eine Anhebung in drei Stufen durchgesetzt. [Artikel im Internet]. Verfügbar unter: https:// www.spiegel.de/wirtschaft/tabaksteuer-erhoehung-regierung-will-raucher-bei-der-stange-halten-a-250588.html [04.11.2019].

SUCHT (2020). Deklaration konkurrierender Interessen /Declaration of competing interests. [Dokument im Internet]. Verfügbar unter: https://www.hogrefe.com/index.php?elD=dumpFile\&t=f $\& f=4431 \&$ token $=52 a 7 b 8559 b 2 c 247 d b 609$ e $4130 a 72 e b b 230 b$ $3 c b 28[15.02 .2021]$.

Süddeutsche Zeitung (2021). Scholz vernebelt die TabaksteuerErhöhung. [Artikel im Internet]. Verfügbar unter: https://www. sueddeutsche.de/gesundheit/tabaksteuer-rauchen-zigaret ten-public-health-praevention-krebs-1.5204705 [15.02.20 21].

The PLoS Medicine Editors (2008). Making Sense of Non-Financial Competing Interests. PLoS Med 5(9): e199. https://doi. org/10.1371/journal.pmed.0050199

WHO (nach 2004). Tobacco Explained - The truth about the tobacco industry ... in its own words. [Dokument auf Webseite]. Verfügbar unter: https://www.who.int/tobacco/media/en/TobaccoExplained.pdf [16.09. 2019].

\section{Historie}

Manuskript eingereicht: 17.02.2021

Manuskript angenommen: 25.02.2021

\section{Deklaration konkurrierender Interessen}

\section{Prof. Dr. Gerhard Bühringer}

Direkte Interessen: Die finanzielle Förderung (alleine oder zusammen mit anderen Projektleitern) für Forschungsvorhaben zum Glücksspielen (Spieler- und Verbraucherschutz, Regulierungskonzepte) am IFT Institut für Therapieforschung bzw. an der TU Dresden erfolgte in den letzten 15 Jahren durch folgende Organisationen: Bayerisches Staatsministerium für Gesundheit und Pflege aus Mitteln des Bayerischen Staatsministeriums der Finanzen, für Landesentwicklung und Heimat (oberste Glücksspielaufsichtsbehörde über eigene Glücksspielangebote im Rahmen des Glücksspielmonopols sowie über private Anbieter), Bundesministerium für Gesundheit, Bundesministerium für Wirtschaft (Aufsichtsbehörde über Teilbereiche des Rechts für Geldspielautomaten), Deutsche Forschungsgemeinschaft (Einzelförderung sowie SFB-Teilprojekt), Mitglieder des Düsseldorfer Kreis (Westdeutsche Lotterie und Lotto Thüringen, Westdeutsche Spielbanken und Spielbank Bad Homburg, Tipico, Löwen Entertainment und Schmidt Gruppe); Europäische Kommission (ALICERAP) sowie weitere staatliche und gewerbliche Glücksspielanbieter (b-win, Baden-Württembergische Spielbanken,

Bundesverband deutscher Spielbanken).

Förderung einer multizentrischen internationalen Psychotherapiestudie zur Behandlung älterer Alkoholabhängiger durch die Lundbeck Stiftung und eines Therapiekonzepts für die Behandlung psychischer Störungen bei Piloten durch insgesamt 11 Fluggesellschaften. Die fördernden Stellen hatten keinen Einfluss auf Untersuchungsplan, Durchführung, Auswertung, Interpretation und Publikation der Studien und ihrer Ergebnisse.

Indirekte Interessen: Mitgliedschaft im Düsseldorfer Kreis: interdisziplinäre Initiative von Vertretern der öffentlichen und privaten Glücksspielanbieter, der Forschung, Beratung und des Hilfesystems zur Entwicklung von Vorschlägen für eine bundesweite Glücksspielregulierung sowie für einen öffentlich kontrollierten Jugend-, Verbraucher- und Spielerschutzes (www.duesseldorferkreis.de). Weiterhin 2008-2019 Mitglied und früherer Vorsitzender des Wissenschaftlichen Beirat der Europäischen Drogenbeobachtungsbehörde (EMCDDA), der u.a. Verbotsempfehlungen und Forschungsempfehlungen für die Europäische Kommission erarbeitet.

\section{Robert Czernecka}

Die Forschungsarbeiten von Robert Czernecka werden teilweise durch eine unbeschränkte Zuwendung von Mitgliedern des Düsseldorfer Kreises an die TU Dresden unterstützt.

\section{Förderung}

Open-Access-Veröffentlichung ermöglicht durch SLUB Dresden.

\section{Prof. Dr. Gerhard Bühringer}

Institut für Klinische Psychologie und Psychotherapie Technische Universität Dresden

Chemnitzer Str. 46

01087 Dresden

gerhard.buehringer@tu-dresden.de 